\title{
Variability of response to the fluid bolus is again demonstrated
}

\author{
Ellis R. Muggleton * (D) \\ See related research by Obonyo et al. https://ccforum.biomedcentral.com/articles/10.1186/s13054-017-1679-0
}

The AFRIM study by Obonyo et al. [1] presents haemodynamic data worthy of closer analysis. The inferior vena cava collapsability index (IVCCI) was used as a surrogate measure of intravascular volume status. However, this measurement is of limited use in spontaneously breathing patients, as demonstrated by a recent meta-analysis [2]. According to Table 1 from [1], 100\% of group 1 and group 2 demonstrated chest indrawing leading to the generation of significant negative intrathoracic pressures and making the measurements invalid.

The purpose of a fluid bolus is to increase stroke volume and hence cardiac output. Although these patients were dehydrated, only 5 out of 11 in group 1 and 5 out of 9 in group 2 demonstrated a $10 \%$ increase in stroke volume following the fluid boli. Two patients in group 1 demonstrated a $10 \%$ decrease in stroke volume index, with a decrease of $48 \%$ in one case! It would interest us what the authors believe the reason for this effect is.

We believe that the administration of fluid, even in hypovolaemic patients, is more complex than often appreciated, with an interaction of many factors related to vascular biology, and further investigation is required to enable an optimisation of fluid delivery and to avoid the detrimental effects of fluid overload.

\section{Authors' response}

The AFRIM study authors

We are encouraged by the interest in fluid resuscitation in children with severe acute malnutrition and hypovolaemic shock, and have read with keen interest the contribution by Dr. Ellis Muggleton highlighting the limited utility of IVCCI in spontaneously breathing patients.

In the manuscript, while presenting these data [1], the authors noted the limitations in interpretation of the IVCCI, especially with respiratory distress characterised by deep (Kussmaul's) breathing and chest indrawing. Given the limited ability of IVCCI to predict fluid responsiveness (FR), it is important to underline the fact that a single negative test with recognised limited utility cannot be used to rule out FR [2] and more research on non-invasive assessment of FR is needed as there is still emerging research advocating utility of IVCCI [3].

Reduction in stroke volume index following fluid bolus administration is indeed an unexpected finding. Nonetheless, our findings stimulate a new direction of inquiry investigating the mechanisms underlying the pathophysiology of hypovolaemia with severe malnutrition as well as the interpretation of the patients' relative position on the Frank-Starling curve with respect to FR. This is particularly important in the context of little understanding on the effect that fluid administration has on the complex patho-biological interaction of intravascular hypovolaemia and severe malnutrition. Previous research has shown that hypovolaemia and dehydration are associated with higher mortality in severe malnutrition [4]. The AFRIM study showed no supportive evidence of increased risk of cardiac failure in severely malnourished children receiving fluid resuscitation for hypovolaemic shock [1]. The patient with a stroke volume reduction of $48 \%$ also had an increase in the systemic vascular resistance index of $56 \%$, which could be indicative of an advanced stage of illness with extreme physiological compensation. 


\section{Abbreviations}

FR: Fluid responsiveness; IVCCI: Inferior vena cava collapsability index

\section{Acknowledgements}

None.

Funding

None.

Availability of data and materials

Not applicable.

\section{Author's contribution}

ERM wrote the manuscript.

Ethics approval and consent to participate

Not applicable.

\section{Consent for publication}

Not applicable.

\section{Competing interests}

The author declares that she has no competing interests.

\section{Publisher's Note}

Springer Nature remains neutral with regard to jurisdictional claims in published maps and institutional affiliations.

Published online: 25 August 2017

\section{References}

1. Obonyo N, Brent B, Olupot-Olupot P, et al. Myocardial and haemodynamic responses to two fluid regimens in African children with severe malnutrition and hypovolaemic shock (AFRIM study). Crit Care. 2017;21:103.

2. Long E, Oakley E, Duke T, Babl FE. Does respiratory variation in inferior vena cava diameter predict fluid responsiveness: a systematic review and meta-analysis. Shock. 2017:47(5):550-9.

3. Corl KA, George NR, Romanoff J, Levinson AT, Chheng DB, Merchant RC, Levy MM, Napoli AM. Inferior vena cava collapsibility detects fluid responsiveness among spontaneously breathing critically ill patients. J Crit Care. 2017;41:130-7.

4. Talbert A, Thuo N, Karisa J, Chesaro C, Ohuma E, Ignas J, Berkley JA, Toromo C, Atkinson S, Maitland K. Diarrhoea complicating severe acute malnutrition in Kenyan children: a prospective descriptive study of risk factors and outcome. PLoS One. 2012;7(6):e38321. 